\title{
Ombro flutuante associado à luxação escápulo-umeral posterior: relato de caso*
}

\author{
Floating shoulder combined with \\ posterior scapulohumeral dislocation
}

\begin{abstract}
Alessandro Ulhôa Rodrigues', Marco Antonio de Castro Veado², Gustavus Lemos Ribeiro Melo³
\end{abstract}
\section{RESUMO}

Apresenta-se rara variação de ombro flutuante associada a luxação glenoumeral posterior em paciente masculino, 26 anos de idade, vítima de acidente motociclístico. Ao exame, queixava-se de dor no ombro esquerdo, limitação dos movimentos, principalmente da rotação externa. A radiografia em ântero-posterior (AP) verdadeiro mostrou fratura do colo da glenóide, sem envolvimento articular, e fratura do processo coracóide. A radiografia em perfil, axilar e a tomografia computadorizada confirmaram a luxação posterior. Os autores dis-

* Trabalho realizado no Serviço de Ortopedia e Traumatologia do Hospital Mater Dei, Belo Horizonte (MG), Brasil.

1. Cirurgião do Ombro e Cotovelo do Hospital Mater Dei, Belo Horizonte (MG), Brasil.

2. Professor da Faculdade de Ciências Médicas de Minas Gerais FCMMG; Cirurgião do Ombro e Cotovelo do Hospital Mater Dei, Belo Horizonte (MG), Brasil.

3. Médico Residente (R2) em Ortopedia e Traumatologia do Hospital Mater Dei, Belo Horizonte (MG), Brasil.

Endereço para correspondência: Rua Rio Grande do Norte, 726, 15o andar, Funcionários - 30130-131 - Belo Horizonte (MG), Brasil. E-mail: castrove@ terra.com.br

Recebido em 3/3/07. Aprovado para publicação em 30/10/07.

Copyright RBO2007 cutem as questões que fazem deste um caso singular no qual se optou por redução aberta e fixação da fratura com placa de reconstrução pélvica. $O$ controle radiológico confirmou o restabelecimento da anatomia. A fratura consolidou-se, resultando em um ombro normal e funcional.
Descritores - Ombro/patologia; Ombro/lesões; Luxação do ombro/diagnóstico; Fraturas do ombro; Re- latos de casos [Tipo de publicação]

\section{ABSTRACT}

$A$ rare variation of the floating shoulder is presented, combined with a posterior glenohumeral dislocation in a male, 26 year old patient, victim of a motorcycle accident. On physical examination, he complained of pain in the left shoulder, limitation of should movements, in particular external rotation. True anteroposterior $X$-ray showed a fracture of the glenoid neck without joint involvement and fracture of the coracoid process. Profile, axillary X-ray and CT scan confirmed the posterior dislocation. The authors discuss the features that make this case a unique case for which open reduction and fracture fixation with a pelvic reconstruction plate were chosen. Radiological follow-up confirmed recovery of the anatomy. The fracture healed and the result is a normal, functional shoulder.
Keywords - Shoulder/pathology; $\quad$ Shoulder/injuries; Shoulder dislocation/diagnosis; Shoulder fractures; Case reports [Publication type]




\section{INTRODUÇÃO}

O "ombro flutuante" é uma afecção pouco freqüente, causada por trauma de alta energia e definida como uma dupla ruptura do complexo suspensor superior do ombro. Geralmente, ocorre uma fratura ipsilateral do colo da glenóide associada a uma fratura da diáfise da clavícula e ruptura dos respectivos ligamentos. A literatura é controversa em relação ao tratamento. Apresentamos rara variação dessa afecção, em que o paciente era portador de fratura ipsilateral do colo da glenóide e da base do processo coracóide acompanhada de luxação posterior da articulação glenoumeral.

\section{RELATO DO CASO}

Paciente masculino, 26 anos de idade, leucodérmico, publicitário, destro, vítima de acidente motociclístico com trauma direto no ombro esquerdo. Atendido inicialmente em outro hospital com diagnóstico de fratura da escápula esquerda, encaminhado para "continuidade do tratamento conservador da fratura".

Ao exame, apresentou dor forte no ombro esquerdo, importante limitação dos movimentos da articulação glenoumeral, principalmente da rotação externa, escoriação na região posterior do ombro, edema e deformidade sugerindo luxação posterior da articulação glenoumeral esquerda. Pulso preservado e sem déficit neurológico da extremidade afetada. A série trauma foi feita; a incidência em axilar foi somente realizada posteriormente com o paciente já anestesiado. A radiografia em AP verdadeiro confirmou a fratura do colo da glenóide sem envolvimento articular e uma fratura do processo coracóide, caracterizando uma variação de ombro flutuante. O perfil da escápula sugeriu luxação glenoumeral posterior (figura 1).

A tomografia computadorizada (TC) confirmou a fratura extra-articular do colo da glenóide, que se encontrava totalmente anteriorizada, fratura da base do processo coracóide, associadas à luxação posterior da cabeça umeral (figuras 2a, 2b e 2c).

No centro cirúrgico, sob bloqueio interescaleno e sedação, não foi possível obter-se a redução da luxação, mesmo após várias tentativas. A visualização fluoroscópica revelou que a tração era ineficiente, pois estava havendo um movimento anormal no foco da fratura, impossibilitando, assim, a redução incruenta.

Programaram-se, então, a redução cruenta da luxação e o tratamento cirúrgico da fratura do colo da glenóide.

O paciente foi posicionado em decúbito lateral direito, sob anestesia geral e bloqueio interescaleno, com o membro superior esquerdo (MSE) livre para movimentação durante o ato cirúrgico.

Feita incisão longitudinal de $10 \mathrm{~cm}$ na região posterior do ombro esquerdo, tipo Judet, na junção dos dois terços mediais com o terço lateral da espinha da escápula. O deltóide posterior foi afastado superiormente, facilitado pela abdução do braço. Identificação do espaço entre o músculo infra-espinhal e o redondo menor com evidenciação do foco da fratura, que se iniciava na incisura escapular e descia pelo colo, até terminar na borda medial da escápula. O nervo supra-escapular estava íntegro, porém pinçado no foco da fratura.

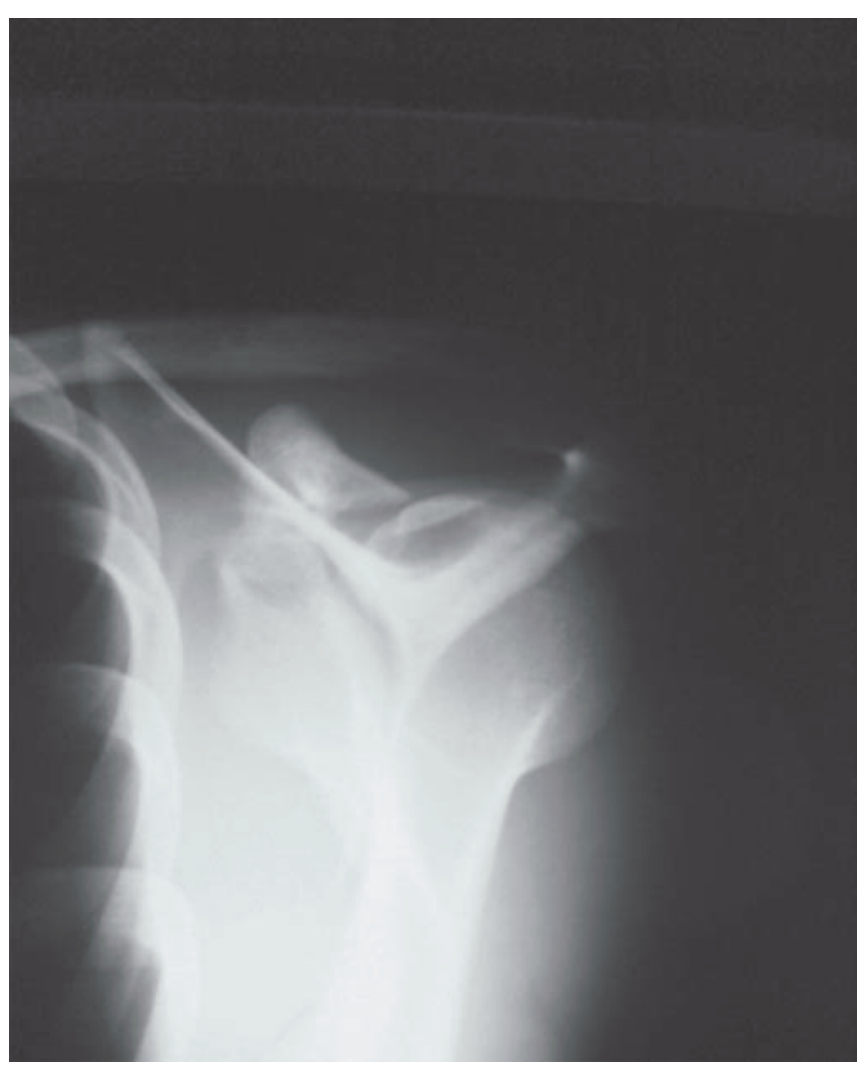

Figura 1 - Perfil escapular sugerindo luxação posterior, mostrando fratura da base do processo coracóide 


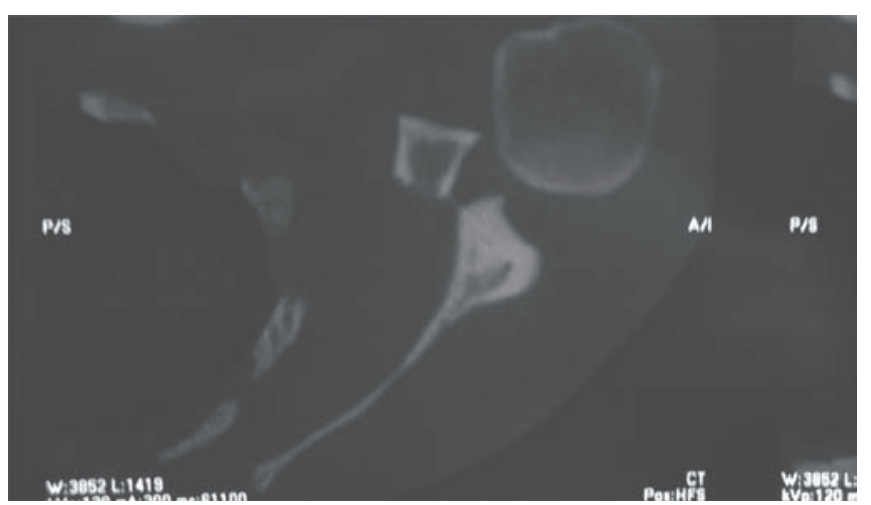

Figura 2a - Tomografia computadorizada evidenciando fratura da glenóide. Observar cabeça umeral luxada posteriormente.

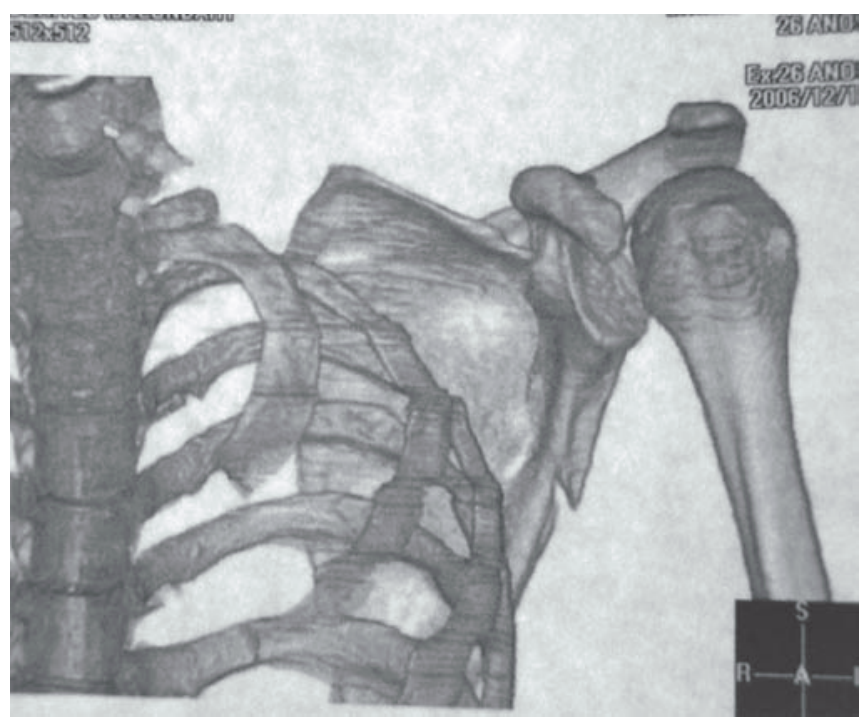

Figura 2b - Reconstrução tridimensional da TC

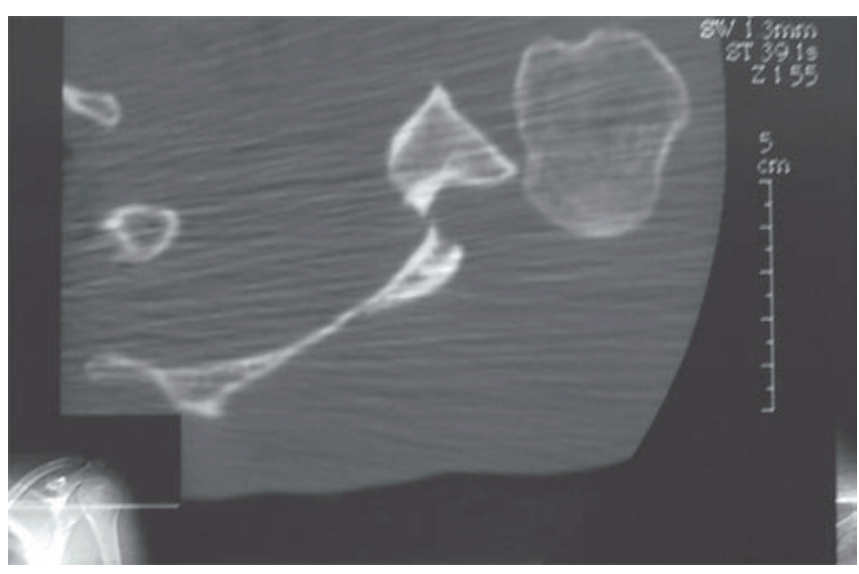

Figura 2c - TC após manipulação no centro cirúrgico: insucesso na redução.

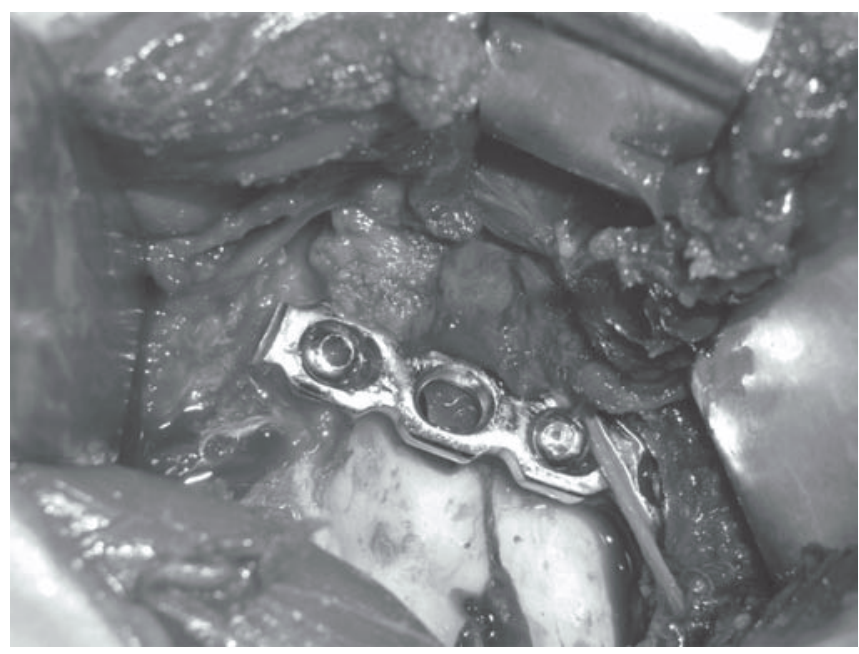

Figura 3a - Fixação da fratura do colo da glenóide com placa de reconstrução acetabular. Observar o nervo supra-escapular atravessando o foco da fratura (foi protegido para evitar seu contato com a placa).

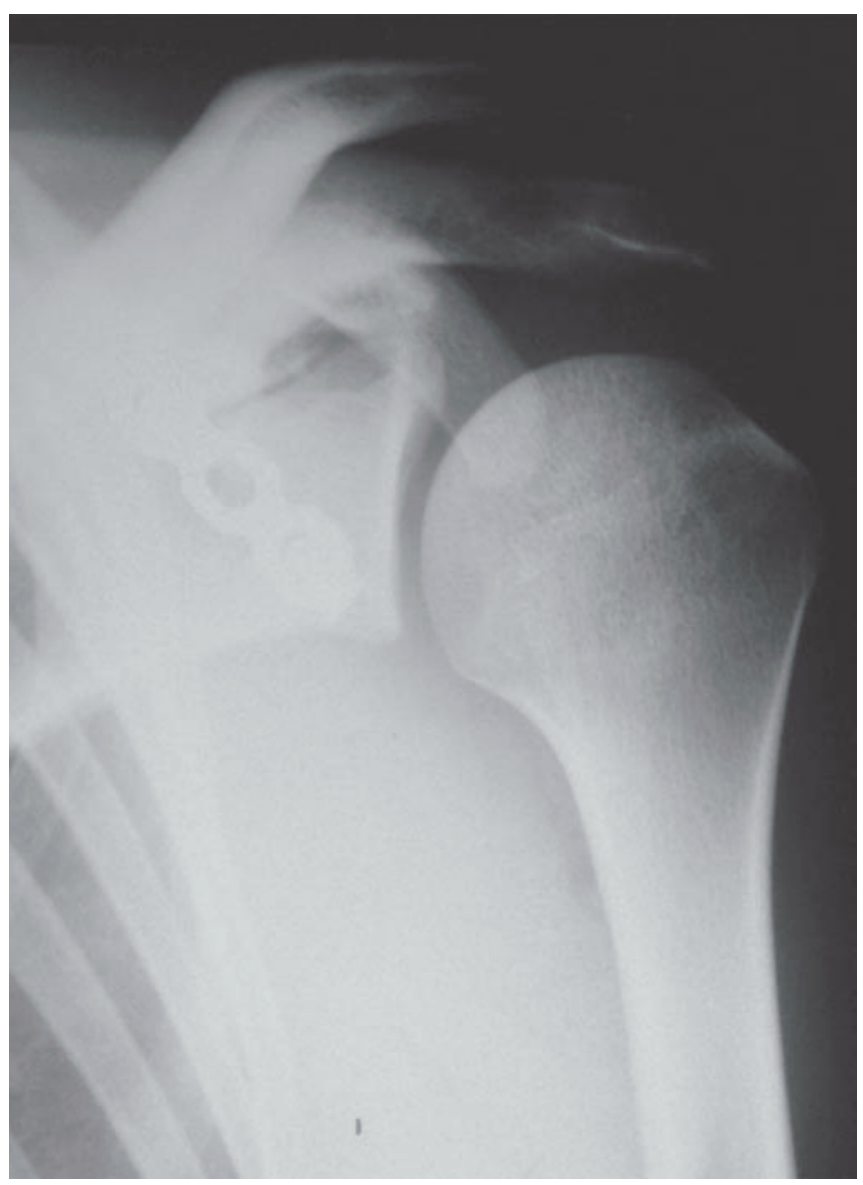

Figura 3b - Radiografia em AP do ombro pós-operatório: fratura do colo da glenóide fixada com placa pélvica 


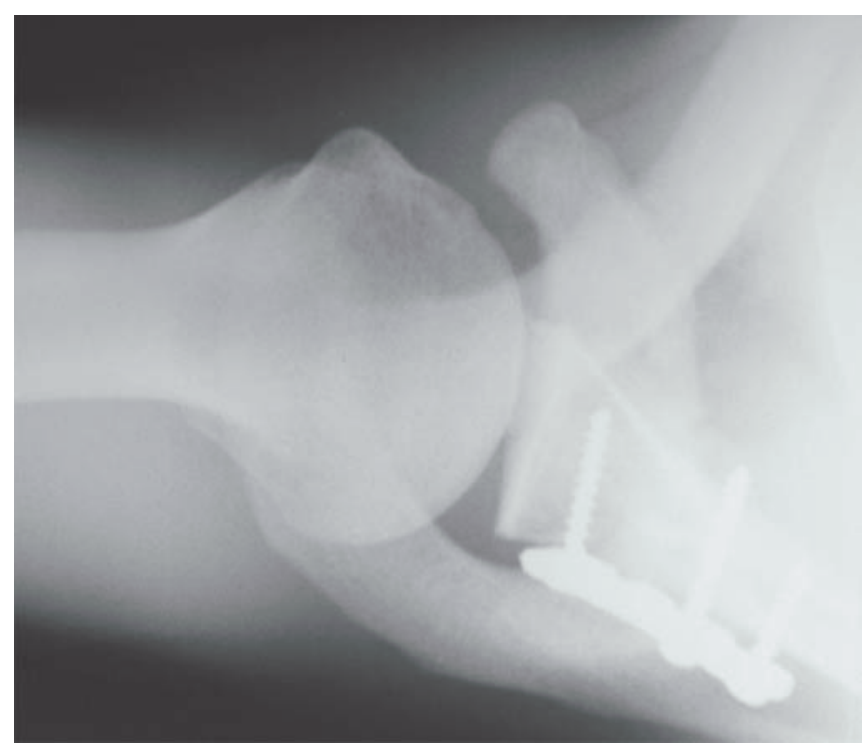

Figura 3c - Radiografia em axilar pós-operatório: boa redução.

A luxação foi reduzida sem muita dificuldade e a cápsula posterior foi aberta para visualização da articulação.

A fratura foi reduzida e fixada com uma placa de reconstrução pélvica com somente três parafusos corticais em função da posição da fratura, após prévio afastamento do nervo supra-escapular (figuras $3 \mathrm{a}, 3 \mathrm{~b}$, $3 c)$.

Qualquer bloqueio mecânico foi afastado após verificação de ampla movimentação articular.

O controle radiológico confirmou a redução da luxação glenoumeral, uma osteossíntese satisfatória da fratura do colo da glenóide e redução da fratura da base do processo coracóide (que não necessitou fixação), reconstruindo, assim, a anatomia do ombro.

O paciente foi imobilizado em tipóia tipo velpeau, pois a redução da articulação mostrava-se estável, mesmo na posição de rotação interna máxima. Durante a evolução, a consolidação se deu normalmente, resultando em um ombro funcionalmente normal.

\section{DISCUSSÃO}

O ombro flutuante (OF) foi descrito inicialmente por Ganz et al em 1975, como uma rara lesão, que consistia de fratura do colo da glenóide associada a fratura ipsilateral da diáfise da clavícula ${ }^{(1)}$. Herscovici et al relataram incidência de $0,1 \%$ de of nos traumas da

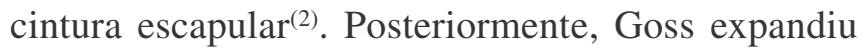
essa definição e descreveu a "dupla ruptura" do complexo suspensor superior do ombro (CSSO) $)^{(3)}$. É de suma importância, para se entender, diagnosticar e tratar essa afecção, bom conhecimento da anatomia do CSSO, que, segundo Owens et al, é um anel formado por partes moles (ligamentos) e osso, ligado ao tronco pelo qual o membro superior é sustentado(4). Esses autores subdividiram esse complexo em três unidades: I) clavícula-articulação acromioclavicular II) acrômio-processo coracóide-colo da glenóide fixados ao corpo da escápula; III) clavícula-ligamentos coracoclavicularesprocesso coracóide ${ }^{(4)}$.

Apresentamos rara variação do II subtipo, ainda não encontrada na literatura pesquisada.

O paciente apresentou fratura do colo da glenóide desviada anteriormente, com fratura da base do processo coracóide e luxação da articulação glenoumeral posterior.

O conceito de dupla ruptura do CSSO permite-nos compreender a variedade de lesões que ocorre no ombro, que, quando analisadas separadamente, têm um comportamento, mas quando juntas, têm uma biomecânica única.

Isoladamente, cada fratura pode apresentar pequeno desvio e ser tratada conservadoramente. Por outro lado, quando combinadas, uma fratura pode aumentar o desvio da outra. A resultante instabilidade deverá aumentar se adicional ruptura dos respectivos ligamentos estiver presente. Nesta eventualidade, a lesão torna-se instável, podendo resultar em falha de consolidação das fraturas, determinando impacto subacromial, fraqueza e desconforto na cintura escapular ${ }^{(5)}$.

Williams et al, em estudos biomecânicos em cadáveres, determinaram que fraturas associadas ipsilaterais da diáfise da clavícula e do colo da glenóide, sem ruptura dos ligamentos coracoclaviculares, não eram suficientes para determinar quadro de ombro flutuante ${ }^{(6)}$.

Por se tratar de afecção rara, a literatura ainda é controversa em relação ao diagnóstico e, principalmente, em relação ao tratamento. 
Herscovici et al indicam tratamento cirúrgico somente para clavícula, quando esta ocorre associada a fratura do colo da glenóide( ${ }^{(2)}$.

Ramos et al relataram bons resultados em 19 pacientes com of tratados não cirurgicamente ${ }^{(7)}$. Maris et al recomendam a fixação de ambas as fraturas da clavícula e colo da glenóide, quando ocorre desvio significante ${ }^{(8)}$.

Giordano et al indicam tratamento cirúrgico para fraturas do colo da glenóide se há angulação maior do que $40^{\circ}$, medialização da glenóide maior do que $2 \mathrm{~cm}$ e fratura articular desviada ${ }^{(9)}$.

O mecanismo do trauma descrito para produzir quadro de of é normalmente o de alta energia, sendo principalmente acidentes automobilísticos e motociclísticos ou queda de altura. No caso em questão, temos essa hipótese confirmada, já que o paciente sofreu trauma direto após queda de motocicleta.

Os objetivos do tratamento foram a redução da luxação, a execução de osteossíntese rígida da fratura, a preservação da mobilidade da articulação glenoumeral e restauração da função global do ombro. $\mathrm{O}$ tratamento pode ser considerado um desafio para o cirurgião, a partir do momento em que estamos diante de exceções. Observamos que o melhor posicionamento do paciente para o ato cirúrgico era o decúbito lateral, pois conseguimos mobilizar livremente o braço, facilitando a redução da fratura.

Há quem preconize posicionar o paciente em decúbito ventral ${ }^{(10)}$, o que nos pareceu menos vantajoso, pois, com a abdução do ombro, conseguimos afastar o deltóide superiormente, não sendo necessário divulsionar as suas fibras. A via de acesso posterior preconizada por Judet, como descrita por Rowe, parece ser a melhor opção, pois permite ampla exposição cirúrgica, inclusive quando há necessidade de abertura da articulação ${ }^{(11)}$. É imperativo o bom conhecimento da anatomia para preservação do nervo supra-escapular, pois este pode ser facilmente lesado após dissecção do músculo infra-espinhal.

No caso aqui relatado, a fratura foi reduzida com certa facilidade, mas encontramos dificuldades na colocação da placa, pois a escápula é um osso chato com poucos locais para introdução dos parafusos. Obser- vamos que, com a redução da luxação posterior e da fratura do colo da glenóide, a fratura do processo coracóide reduziu-se sem qualquer manobra, não havendo necessidade de fixá-la. A indicação do tratamento cirúrgico se impôs devido à instabilidade própria do ombro flutuante e à associação com a luxação posterior, que não era passível de ser reduzida incruentamente. Além disso, tratava-se de paciente jovem, com boa qualidade óssea e sem lesão prévia nesse ombro.

O rápido reconhecimento da lesão facilitou a redução da fratura e da luxação durante o ato cirúrgico, resultando em um ombro funcional e estável, o paciente apresentando força muscular normal (M5), retorno às suas atividades esportivas anteriores ao trauma e ótima amplitude de movimento, sendo a elevação anterior a $180^{\circ}$, rotação externa de $70^{\circ}$ e rotação interna em T7.

\section{REFERÊNCIAS}

1. Ganz R, Noesberger B. [Treatment of scapular fractures]. Hefte Unfallheilkd. 1975;(126):59-62. German.

2. Herscovici D Jr, Fiennes AG, Allgöwer M, Rüedi TP. The floating shoulder: ipsilateral clavicle and scapular neck fractures. J Bone Joint Surg Br. 1992;74(3):362-4. Comment in: J Bone Joint Surg Br. 1993;75(3):509.

3. Goss TP. Double disruptions of the superior shoulder suspensory complex. J Orthop Trauma. 1993;7(2):99-106.

4. Owens BD, Goss TP. The floating shoulder. J Bone Joint Surg Br. 2006;88(11):1419-24. Comment in: J Bone Joint Surg Br. 2007;89(5):708; author reply 708 .

5. Goss TP. Scapular Fractures and Dislocations: Diagnosis and Treatment. J Am Acad Orthop Surg. 1995;3(1):22-33.

6. Williams GR Jr, Naranja J, Klimkiewicz J, Karduna A, Iannotti JP, Ramsey M. The floating shoulder: a biomechanical basis for classification and management. J Bone Joint Surg Am. 2001; 83-A(8):1182-7.

7. Ramos L, Mencía R, Alonso A, Ferrández L. Conservative treatment of ipsilateral fractures of the scapula and clavicle. J Trauma. 1997;42(2):239-42.

8. Maris JS, Papanikolaou A, Karadimas E, Petroutsas JA, Karabalis C, Deimedes G, Tsampazis K. The management of combined fractures of the clavicle and the scapular neck (floating shoulder). J Bone Joint Surg Br. 2003;85-B(Suppl 3):212.

9. Giordano G, Accabled F, Besombes C, Tricoire JL, Chiron P. Floating shoulder: orthopaedic and surgical management of a series of 45 patients. J Bone Joint Surg Br. 2004;86-B(Suppl 1):64.

10. Michael D, Fazal MA, Cohen B. Nonunion of a fracture of the body of the scapula: case report and literature review. J Shoulder Elbow Surg. 2001;10(4):385-6.

11. Rowe T. Surgical approaches to the shoulder. In: Rowe CR, editor. The shoulder. New York: Churchill Livingstone; 1988. p. $35-51$. 